\title{
Problèmes posés par la réfrigération des centrales de grande puissance situées sur le littoral
}

\author{
par J.-C. Lebreton \\ Chef du Département Etudes Générales, Programmes, Sites, Environnement \\ Direction de l'Equipement d'Electricité de France
}

\section{Introduction et définitions}

Quoique constituant des généralités de mieux en mieux connues, nous rappellerons un certain nombre de faits prospectifs caractérisant le développement de l'énergie électrique au cours des trente prochaines années.

En fin de siècle, la consommation annuelle devrait être proche de $1000 \mathrm{TWh}$, correspondant à une puissance appelée à la pointe de $175 \mathrm{GW}$. Il faudra disposer alors d'une puissance installée de $200 \mathrm{GW}$, dont 35 seraient des moyens de production de pointe et $165 \mathrm{GW}$ des moyens de base.

La production de base sera assurée en totalité ou presque par des centrales nucléaires nouvelles et, si l'on estime à $4 \%$ la progression de la consommation en l'an 2000, ce qui implique la mise en construction de $35 \mathrm{GW}$ à cette date, on constate qu'il faut mettre en chantier entre 1974 et $2000: 165+35$, soit $200 \mathrm{GW}$, moins les $15 \mathrm{GW}$ actuellement en construction, soit au minimum $185 \mathrm{GW}$. Ceci est bien un minimum puisqu'il n'a pas été tenu compte des arrêts progressifs des centrales thermiques ou nucléaires ayant plus de vingt ou trente années de service.

En prenant une valeur moyenne pour la puissance implantable pour chaque site, on trouverait le nombre minimum de sites à ouvrir. Le choix d'une telle valeur peut résulter de considérations diverses:

- modulation des puissances installées en fonction de la répartition des centres de consommation;

- limitation ou renforcement du réseau de grand transport;

- limitation des modifications apportées à l'environnement.

Ce choix s'avère extrêmement complexe, et aucun élément décisif ne permet d'avancer aujourd'hui des chiffres certains et valables en toute circonstance. Il est raisonnable cependant de considérer $5 \mathrm{GW}$ comme une puissance implantable dans la majorité des sites qui sont à l'étude.
Aller plus loin résultera en partie d'études comme celles qui seront exposées aujourd'hui.

C'est, en effet, la capacité de réfrigération de la source froide qui constitue le paramètre essentiel pour le dimensionnement des centrales électriques nucléaires, une fois le site d'implantation choisi.

Sur les fleuves et rivières, la réfrigération en circuit ouvert, dans laquelle l'eau qui prélève les calories ne passe qu'une fois dans le condenseur, se trouve limitée par les débits d'étiage; compte tenu de la normalisation des réacteurs et des centrales, il est difficile d'envisager plus de $25 \mathrm{GW}$ au total sans faire appel à la réfrigération en circuit fermé, dans laquelle l'eau qui prélève les calories au condenseur les transfère à l'atmosphère par un échangeur, le réfrigérant atmosphérique, avant de retourner au condenseur.

Le problème est tout autre sur le littoral ou en estuaire, par suite du haut pouvoir de dilution des courants marins. Le circuit ouvert s'impose alors mais son fonctionnement s'avère plus complexe, du fait de l'inversion fréquente des courants dans les mers à marée, ou du caractère très variable des courants de vent sur le littoral méditerranéen.

Très schématiquement, la première question qui se pose revient alors à connaître pendant une période de référence - marée, cycle des courants, ou du vent - l'évolution et la forme des isothermes ou, plus exactement, des courbes d'égal échauffement. En mer peu profonde et bien brassée, ces courbes seront peu différentes sur toute la profondeur, et l'échauffement aura une structure quasi bidimensionnelle dès qu'on s'éloignera du point de rejet, comme l'indiquent les modèles qui seront décrits dans la communication du professeur Curtet.

Vue l'étendue de la zone influencée par le rejet, il serait prohibitif d'implanter la prise d'eau totalement en dehors de cette zone et un recyclage partiel parait inévitable pour certaines configurations des courants. 
Notons au passage l'importance de cette notion de recyclage ou, ce qui revient au même, de renouvellement des masses liquides.

Lorsqu'une masse liquide sort du rejet, elle a, selon le moment choisi, une certaine probabilité d'être réaspirée à la prise après une excursion dans le milieu récepteur plus ou moins longue.

Si l'excursion la plus courte correspond au trajet direct du rejet à la prise d'eau, c'est le recyclage « direct» qui ne se produit que si le courant porteur de la masse d'eau est orienté vers la prise.

L'excursion peut également correspondre à une marée ou plus. Au bout d'un certain temps, d'ailleurs, que l'on peut appeler la «période» des échanges atmosphériques, l'échauffement de la masse d'eau aura baissé de moitié. Le recyclage aura alors moins d'importance.

Dans une mer à marée, le recyclage périodique des masses d'eau est inévitable du fait du va-et-vient des courants de marée. Si ces courants étaient tels que les trajectoires des particules soient fermées, le recyclage des eaux serait permanent et aucun renouvellement des masses liquides pompées ne se produirait. L'échauffement limite résulterait de l'équilibre entre les échanges atmosphériques et l'apport continuel de calories.

En fait, les trajectoires ne se rebouclent jamais exactement et les masses d'eau dérivent lentement.

Il en résulte un renouvellement plus ou moins rapide du volume d'eau, influencé par le rejet et, du même coup, une réduction plus ou moins grande de l'échauffement de ce volume.

Si la dérive était telle que les masses d'eau ne passent qu'une seule fois devant le rejet, il suffirait de connaître la période des échanges atmosphériques, la trajectoire des particules et le coefficient de dilution ou de mélange le long de cette trajectoire, pour avoir l'échauffement en tout point. En général, cependant, le phénomène est plus complexe, car une partie des masses d'eau repasse plusieurs fois devant le rejet et l'échauffement en tout point résultera d'une combinaison des trois processus de convection, de diffusion et d'échange atmosphérique.

\section{Echelles des rejets thermiques}

L'importance des rejets thermiques dépend du rendement du cycle thermodynamique, rendement qui varie légèrement selon la filière des réacteurs nucléaires et selon la température de la source froide : à titre d'exemple, on considérera les deux filières les plus probables actuellement, ce sont :

- les réacteurs à eau légère dont le rendement est voisin de $33 \%$

- les réacteurs à neutrons rapides dont le rendement est voisin de $40 \%$

Nous donnerons, pour ces deux filières, des estimations des rejets lorsque la température de la source froide est à $15{ }^{\circ} \mathrm{C}$.

Pour les premiers, une énergie électrique produite de $1 \mathrm{kWh}$ entraîne le rejet à la source froide de $1650 \mathrm{kcal}$.

Pour les seconds, le même kWh entraîne un rejet d'environ $1250 \mathrm{kcal}$ à la source froide.
L'importance des rejets thermiques, pour un site donné, dépendra finalement de trois éléments:

- le type de réacteur;

- la puissance de chaque réacteur;

- le nombre de réacteurs équipant le site.

Ainsi, le flux thermique correspondant à quatre réacteurs de $1200 \mathrm{MW}$, du type PWR, sera, selon les estimations précédentes, de :

$$
4 \times(1200000 / 3600) \times 1650=2200 \text { thermies } / \mathrm{s}
$$

Le débit d'eau nécessaire pour évacuer ce flux thermique, avec une élévation au condenseur $\Delta \theta$, sera donc:

$$
2200 / \Delta \theta
$$

soit $146 \mathrm{~m}^{3} / \mathrm{s}$ pour $15^{\circ} \mathrm{C}$ au condenseur, et $50 \%$ en: plus si $\Delta \theta=10^{\circ} \mathrm{C}$ soit $220 \mathrm{~m}^{3} / \mathrm{s}$.

Le flux thermique de quatre iéacteurs rapides de $1200 \mathrm{MW}$ serait de l'ordre de 1667 thermies/s, ce qui représente un gáin de $25 \%$ par rapport aux réacteurs à eau légère.

On peut, à titüe d'ordre de granceur, essayer de comparer ces flux thermiques aux flux naturels des courants marins.

Pour un débit de $10^{6} \mathrm{~m}^{3} / \mathrm{s}$, l'une des veines dù Gulf Stream, ayant un échauffement de $1{ }^{\circ} \mathrm{C}$, transporte uri flux de $10^{\circ}$ thermies/s.

On peut également comparer l'énergie thermique, rejetée pendant 24 heures par une centrale de quatre riacteurs de $1,2 \mathrm{GW}$, à l'énergie solaire reçue en movenne par une surface de $100 \mathrm{~km}^{2}$ :

la première est à peu près égale à $30 \%$ de la seconde.

Ces ordres de grandeurs ne doivent être retenus que pour préciser la notion d'échelle dés rejets thermiques de centrales de grande puissance. Ils mettent en valeur l'unique problème que posent de tels rejets: leur dilution dans des volumes de dimension suffisante pour ramener les densités de flux thermiques à l'échelle cies valeurs naturelles. Sans aucune action mécanique, cette difution, résultant du seul processus de diffusion, entrainera des gradients thermiques importants et, par suite, des pics de température aigus. En pratique, heureusement, l'action mécanique des marées ou des vents vient ajouter la contribution essentielle, la convection, et réduit les gradients thermiques dès qu'on s'éloigne de la source.

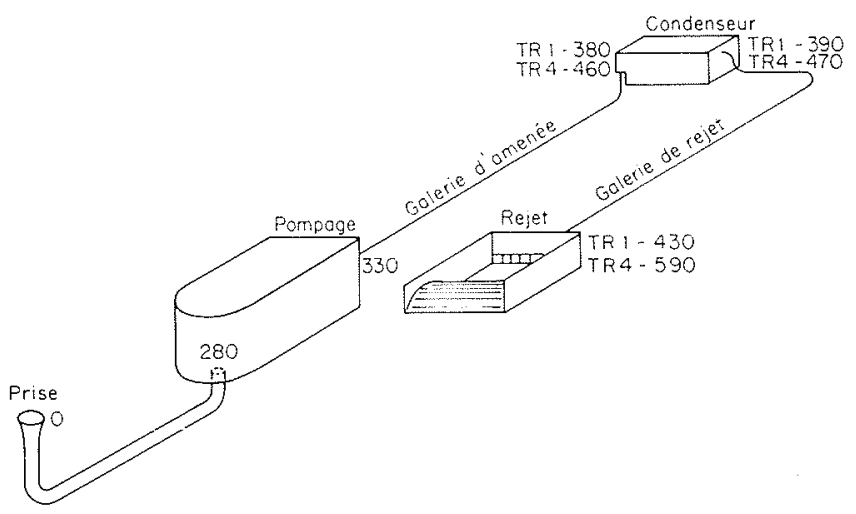

$1 /$ Circuil d'eau d'une centrale implantée sur le littoral de la Manche. 


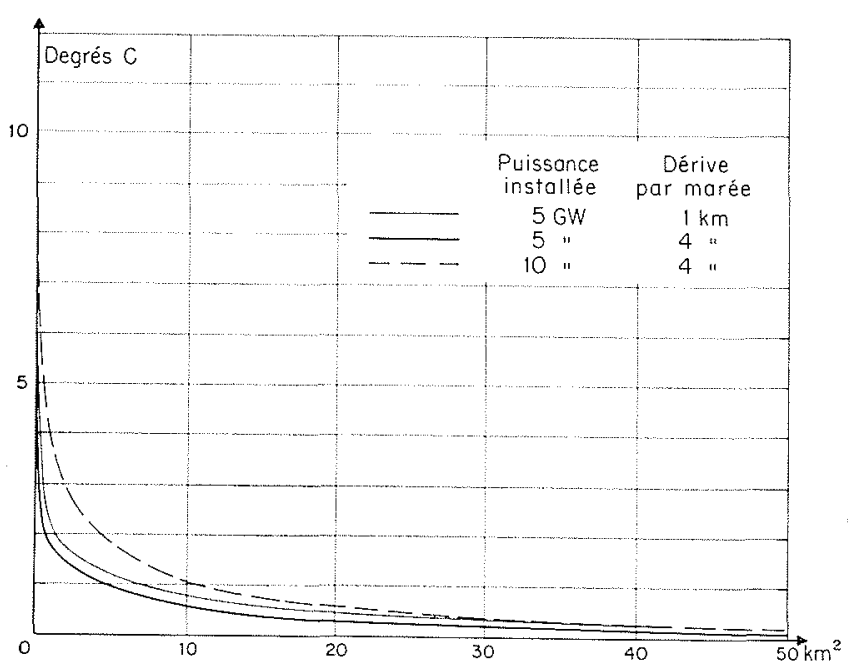

2/ Surfaces échauffées par une centrale thermique sur le site de Paluel.

\section{Différents problèmes résultant de l'échauffement}

Or peut se placer en variable de Lagrange ou en variable d'Euler.

En variable de Lagrange, on suit les particules. Certaines traverseront le circuit d'eau une ou plusieurs fois.

Sur le plan écologique, un certain nombre d'organismes participeront à ce parcours et pourront être affectés par le cycle thermique qu'ils subiront.

Il importe de préciser, non seulement les élévations de température, mais aussi le temps de séjour dans le circuit interne. Pour une centrale actuellement projetée sur le littoral du pays de Caux, le circuit d'eau comporte les éléments successifs suivants (fig. 1):

- galerie d'amenée;

- bassin d'aspiration;

- circuit de pompage;

- condenseur;

- circuit de rejet en conduite;

- parcours dans le canal de rejet.
Les chiffres indiquent le temps de parcours en seconde, compté à partir du passage à travers la prise (instant $t=0$ ).

Il faut donc environ $450 \mathrm{~s}$ à marée haute et $600 \mathrm{~s}$ à marée basse pour parcourir tout le circuit et revenir au milieu marin. Notons cependant que l'élévation de température du condenseur est supportée par la biomasse entraînée pendant une durée n'excédant pas 40 à $90 \mathrm{~s}$, soit $3 \mathrm{mn}$ au maximum à la marée basse.

La plupart des organismes ne suit pas le mouvement des masses d'eau, ce sont les algues, les crustacés et les poissons. L'échauffement "externe» existant dans la zone d'influence de la centrale pourra atteindre ces organismes en permanence. On notera cependant, sur la figure $2^{(a)}$, que ces échauffements ne dépassent $2{ }^{\circ} \mathrm{C}$ que dans une zone rarement supérieure à 500 hectares pour une centrale de $10 \mathrm{GW}$ installée.

Les modifications écologiques qui en résulteront devraient être très limitées dans la plupart des cas.

\section{Conclusion}

Ces quelques réflexions n'ont pour but que de situer les flux thermiques des rejets des centrales nucléaires par rapport à des phénomènes naturels, et surtout de délimiter les principaux problèmes que pose l'intallation des centrales de grancie puissance sur le littoral.

Si les calculs les plus simples sont rassurants, par suite du très grand pouvoir de dilution des courants marins et, en particulier, des courants de marée dans les zones littorales bien «ventilées » (zones ouvertes), il semble indispensable de disposer de modèles assez sûrs pour simuler les situations moyennes et les situations exceptionnelles pouvant survenir dans les états de la mer et à l'atmosphère. Ceci suppose une connaissance statistique solide de l'hydrologie et de la météorologie du littoral à un pas de temps qui peut, sans doute, être pris égal à la période des échanges atmosphériques.

(a) Cette figure est tirée du rapport C 40/74-5, mars 1974, de MM. A. Daubert, J.-F. Malherbe et M. Cazenave.

\section{Discussion}

Président : M. le Professcur A. Fortrer

\footnotetext{
M. le Président remercie M. J.-C. Lebreton pour son exposé qui fixe clairement les ordres de grandeur; il ouvre ensuite la discussion en suggérant de la limiter à des questions se rattachant directement à la communication de M. Lebreton; les problemes strictement techniques seront débattus après les exposés de $M$. Curter et de M. Gauthier.

personne ne demandant la parole, M. le Président présente ensuite les travaux du Comité «Diffusion thermique» de la S.H.F.
} 